\title{
Sigmoid Colonic Stricture Mimicking Hirschsprung Disease in a Cytomegalovirus Positive Immunocompetent Infant
}

\author{
Sohail Ahmad ${ }^{1} \cdot$ Santosh Kumar Mahalik ${ }^{1} \cdot$ Akash Bihari Pati $^{1} \cdot$ Susama Patra ${ }^{2} \cdot$ Kanishka Das $^{1}$
}

Received: 15 October 2020 / Accepted: 5 March 2021 / Published online: 20 April 2021

(C) Dr. K C Chaudhuri Foundation 2021

To the Editor: Cytomegalovirus (CMV) is the commonest intrauterine viral infection with less than forty reported cases of gastrointestinal involvement in the postnatal period $[1,2]$.

A $4.5 \mathrm{~kg}$, 3-mo-old girl presented with recurrent abdominal distension, nonbilious vomiting, and constipation since one month. She was born at term, weighed $2.4 \mathrm{~kg}$ and had passed meconium normally. The abdomen was distended with visible peristalsis. Plain skiagram showed moderate dilation of small bowel, grossly dilated colon, and no rectal air. The contrast enema was unlike Hirschsprung disease, the contrast did not negotiate into the left colon (Supplementary Figure). The leukocyte count was $23,000 / \mathrm{cu} \mathrm{mm}$, thyroid function test and fundoscopy were normal.

Conservative management was unsuccessful. At laparotomy, the sigmoid - descending colon junction showed a short stricture, adjacent friable mucosa and proximal bowel dilatation (Supplementary Figure). The abnormal mucosa was trimmed and stricturoplasty performed. Histopathology showed mucosal ulceration, granulation tissue, and intranuclear CMV inclusions (Supplementary Figure). The postoperative serum CMV - Immunoglobulin M (IgM) was positive but polymerase chain reaction (PCR) for CMV deoxyribonucleic acid (DNA) was negative, hence antiviral therapy was deferred. Fundoscopy and audiometry were normal. She recovered uneventfully and is well after $10 \mathrm{mo}$.

Perinatal CMV transmission is transplacental or postnatal [1]. Infected infants are rarely symptomatic at birth and gastrointestinal involvement is anecdotal in the immunocompetent [3]. Inflammatory colonic strictures in necrotizing enterocolitis of prematurity may feature secondary CMV infection. Only two

Kanishka Das

kanishkadas@hotmail.com

1 Department of Pediatric Surgery, All India Institute of Medical Sciences, Bhubaneswar, Odisha 751019, India

2 Department of Pathology \& Laboratory Medicine, All India Institute of Medical Sciences, Bhubaneswar, Odisha, India term infants with CMV infection and clinical features mimicking Hirschsprung disease have been described [2, 4]. In both, the diagnosis was incidental at histopathology. Several investigations (colorectal endoscopy/laparoscopy, colorectal biopsy) and bowel surgery (colostomy, resection) had punctuated their management before diagnosis. In this case, preoperative evaluation was inconclusive. The inflammatory stricture at laparotomy and CMV inclusions at histopathology clinched the diagnosis. In conclusion, CMV infection may cause sigmoid strictures that mimic rectosigmoid Hirschsprung disease in term, immunocompetent infants.

Supplementary Information The online version contains supplementary material available at https://doi.org/10.1007/s12098-021-03730-8.

\section{Declarations}

Consent to Participate/Publication Written informed consent for publication of clinical details and clinical images was obtained from the parent of the patient. A copy of the consent form is available for review by the Editor of this journal. Obtained from parents.

Conflict of Interest None.

\section{References}

1. Porta A, Avanzini A, Bellini M, et al. Neonatal gastrointestinal involvement and congenital cytomegalovirus. Pediatr Med Chir. 2016;38(3):134.

2. Ekema G, Pedersini P, Milianti S, Ubertazzi M, Minoli D, Manciana A. Colonic stricture mimicking Hirschsprung's disease: a localized cytomegalovirus infection. J Ped Surg. 2006;41:850-2.

3. Tzialla C, Decembrino L, Di Comite A, Bollani L, Colombo R, Stronati M. Colonic stricture and retinitis due to cytomegalovirus infection in an immunocompetent infant. Pediatr Int. 2010;52(4):659-60.

4. Kumar C, Luthra M. Cytomegalovirus as a cause of colonic stricturesimulating Hirschsprung's disease. J Indian Assoc Pediatr Surg. 2018;23:103-5.

Publisher's Note Springer Nature remains neutral with regard to jurisdictional claims in published maps and institutional affiliations. 